\title{
In Situ TEM for Quantitative Electrochemistry of Energy Systems
}

Megan E. Holtz ${ }^{1}$, Yingchao Yu², Johary Rivera ${ }^{2}$, Héctor D. Abruña ${ }^{2}$, and David A. Muller ${ }^{1,3}$

1. School of Applied and Engineering Physics, Cornell University, Ithaca NY 14853.

2. Department of Chemistry and Chemical Biology, Cornell University, Ithaca NY 14853.

${ }^{3 .}$ Kavli Institute at Cornell for Nanoscale Science, Ithaca, NY, USA

In 2010, we began designing a broadly applicable in situ TEM liquid cell chip to enable studies that correlate quantitative electrochemistry with the microstructure of the active material in the TEM. Protochips fabricated the chip we designed, which has demonstrated reliable electrochemical performance even for surface-sensitive measurements such as cyclic voltammetry of fuel cell catalysts, and has become the leading electrochemical cell chip used for quantitative in situ TEM studies today [13]. Here we discuss critical factors behind chip performance, and illustrate applications for alkaline fuel cell studies with an ion exchange membrane, and for lithium ion battery materials.

Through careful materials choices, the in situ chip we designed reproduces the performance of a traditional electrochemical cell (Fig 1). The working electrode is patterned onto the silicon nitride viewing membrane of a liquid flow cell holder (Fig 1ab). For quantitative electrochemistry, the chip must not introduce extraneous electrochemical signals so the process of interest may be studied. We use an electron-transparent glassy carbon working electrode which offers significantly lower scattering than previous metal electrodes. Electrochemically it is cleaner, with a featureless background signal from the capacitive response of the liquid (Fig 1c). The working electrode is entirely in the viewing window, and electrical leads are covered in the photoresist SU8 to prevent additional electrochemical response. We used $\mathrm{Ti}$ instead of $\mathrm{Cr}$ for adhesion layers, to prevent $\mathrm{Cr}$ diffusion that can dominate the electrochemistry. The reference electrode is near the working electrode to minimize uncompensated resistance, and the counter electrode is large and far away to provide ample current and prevent species migration.

Classical test cases for catalyst electrochemistry are quantitatively reproduced in situ, as shown by cyclic voltammetry in $0.1 \mathrm{M} \mathrm{H}_{2} \mathrm{SO}_{4}$ of platinum nanoparticles on Vulcan deposited on the working electrode and measured in the TEM (Fig 1c). This represents a rigorous test case for quantitative electrochemistry, since the features are surface effects that are sensitive to contaminants at the submonolayer level, including hydrogen adsorption and desorption and oxide formation and reduction on the platinum surface. The in situ electrochemistry reproduced the characteristic voltametric profile of the platinum nanoparticles at an appropriate current scale, while a chip with no platinum nanoparticles exhibited only a background current associated with the double layer capacitance of carbon.

Figure 2 shows in situ STEM of platinum nanoparticles separated from the counter electrode by a phosphonium alkaline anion exchange membrane. After flowing in methanol, the cyclic voltammogram displays a methanol oxidation process (Fig 2a). During methanol oxidation, we see formation of particles that are likely carbonates (Fig 2bc), which block pores and poison the fuel cell. The particles are generated by the electrochemistry but assisted in agglomeration by the electron beam - small particles appear over the entire electrode, and only while performing methanol oxidation.

Real time spectroscopic imaging is also possible using valence EFTEM. Fig 3 shows the cycling of a $\mathrm{LiFePO}_{4}$ battery electrode material, where EELS reveals the lithiated and delithiated regions, providing dynamic information about Li-ion transport and degradation of the cathode material $[1,4]$.

\section{References:}


[1] M Holtz et al, NanoLetters 14 (2014) p. 1453.

[2] R Unocic et al, Microscopy and Microanalysis 20 (2014) p. 452.

[3] G.Z. Zhu et al, J. Phys. Chem. C 118 (2014) p. 22111.

[4] Work supported by the Energy Materials Center at Cornell, DOE EFRC BES (DE-SC0001086). EM Facility support from the NSF MRSEC program (DMR 1120296).
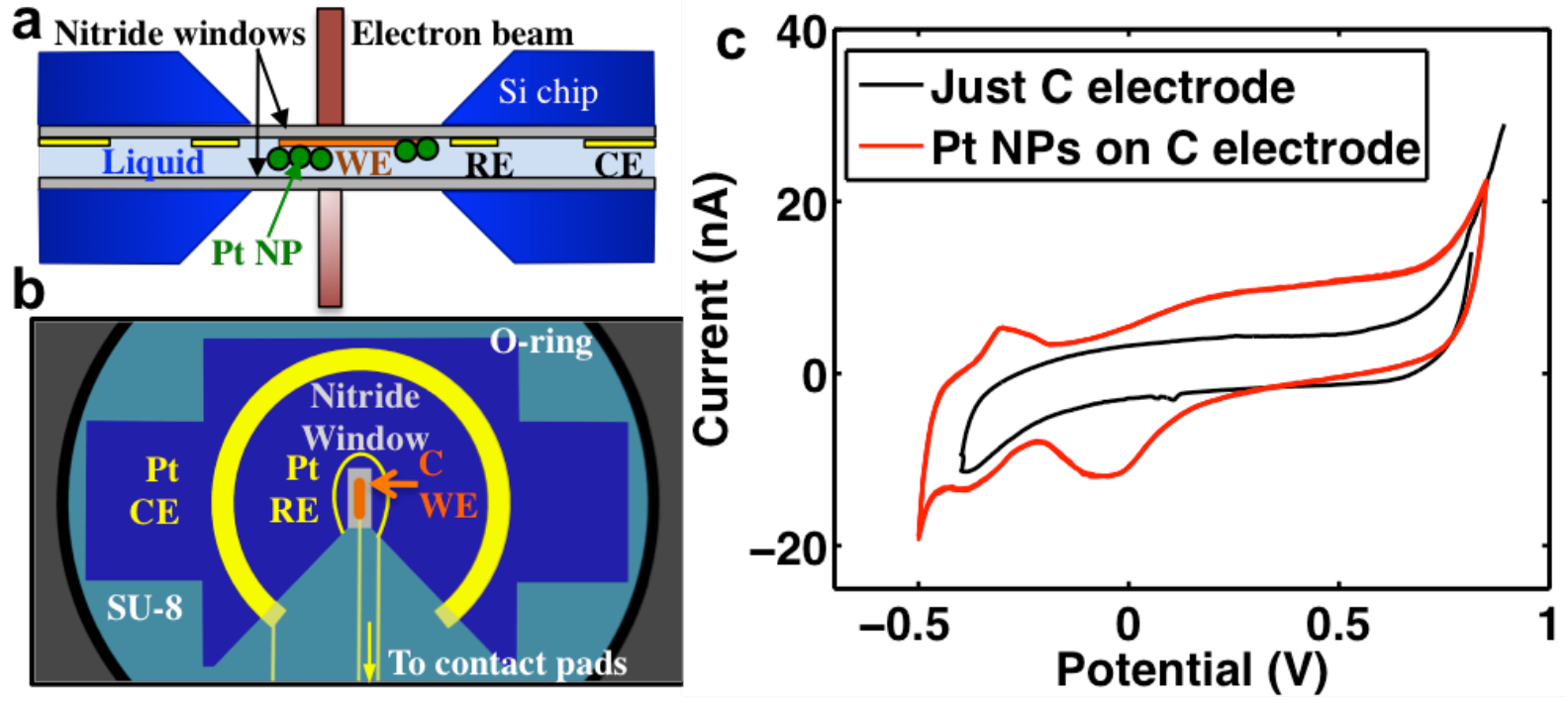

Figure 1. In situ electrochemistry TEM holder and electrochemical data. (a) Cross-section of the chips. (b) Schematic of the top chip, with a carbon working electrode (WE) on the membrane, Pt reference electrode (RE) and counter electrode (CE). The chips exhibited electrochemical activity qualitatively similar to an ex situ microelectrode, as shown for the platinum nanoparticle cyclic voltammetry $(\mathrm{CV})$ in (c). The chip alone with no Pt deposited shows a minimal electrochemical response.

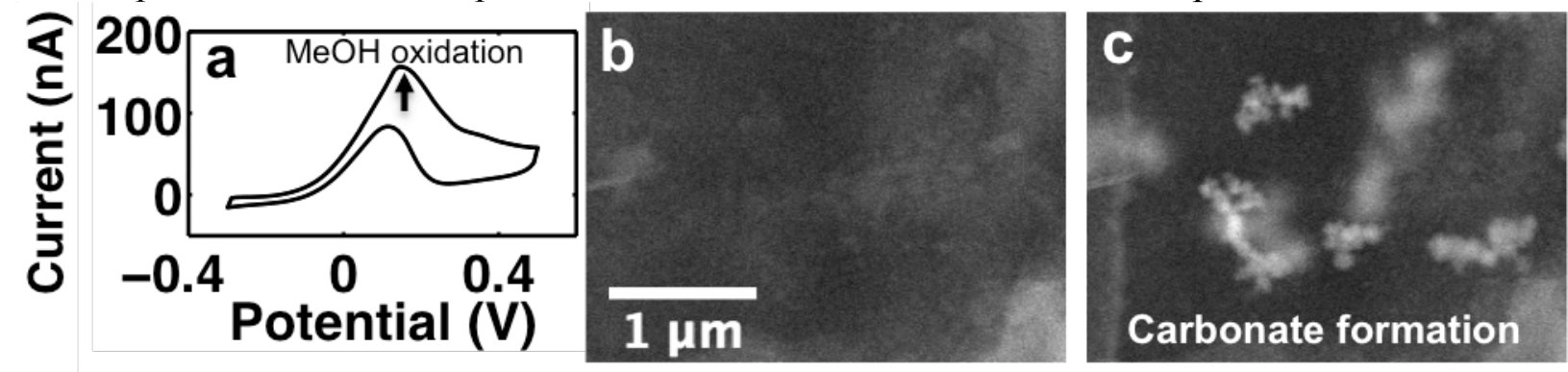

Figure 2. (a) Cyclic voltammogram in $0.1 \mathrm{M} \mathrm{NaOH}$ and $0.1 \mathrm{M}$ methanol with a hydrated phosphonium alkaline anion exchange membrane and Pt catalyst particles in a Tecnai F20 at $200 \mathrm{keV}$. STEM images of (b) before and (c) after methanol oxidation on the membrane. What appear to be carbonate particles appear after cycling. The particles are easily moved and agglomerated under the electron beam.
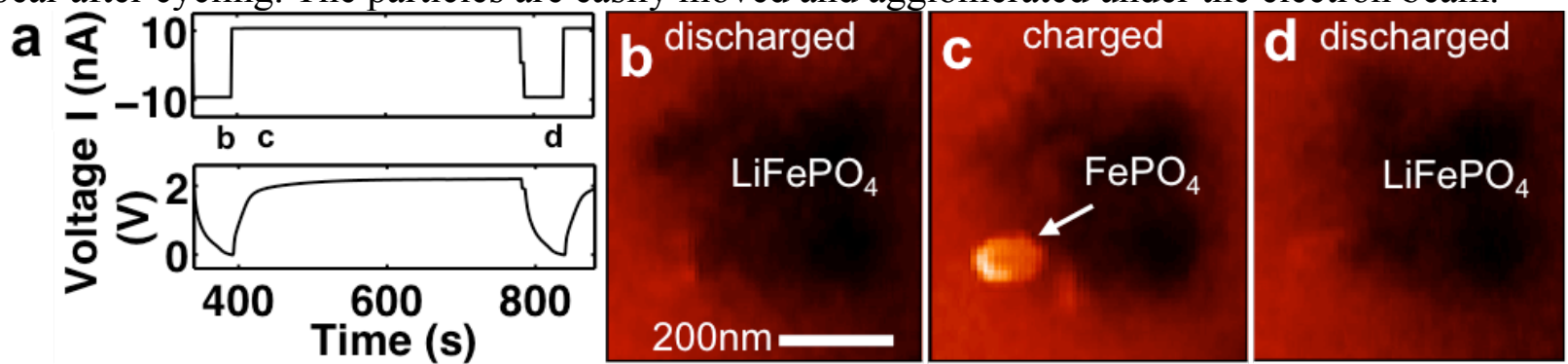

Figure 3. (a) Charge/discharge of the battery cathode material $\mathrm{LiFePO}_{4} .5 \mathrm{eV}$ EFTEM images of the lithiated (b,d) and delithiated (c) $\mathrm{LiFePO}_{4}$, highlighting regions of $\mathrm{FePO}_{4}$, at the times marked in (a). 